\title{
Trois figures antimusicales de la BD franco-belge : la Castafiore, Gaston Lagaffe et Assurancetourix
}

\section{Nicolas Rouvière}

\section{(2) OpenEdition \\ 12 Journals}

Édition électronique

URL : http://journals.openedition.org/recherchestravaux/463

DOI : 10.4000/recherchestravaux.463

ISSN : 1969-6434

Éditeur

UGA Éditions/Université Grenoble Alpes

Édition imprimée

Date de publication : 15 mai 2011

Pagination : 195-212

ISBN : 978-2-84310-200-4

ISSN : 0151-1874

Référence électronique

Nicolas Rouvière, "Trois figures antimusicales de la BD franco-belge : la Castafiore, Gaston Lagaffe et Assurancetourix », Recherches \& Travaux [En ligne], 78| 2011, mis en ligne le 15 novembre 2012,

consulté le 08 septembre 2020. URL : http://journals.openedition.org/recherchestravaux/463 ; DOI : https://doi.org/10.4000/recherchestravaux.463 
Nicolas Rouvière

IUFM de Grenoble

\section{Trois figures antimusicales de la BD franco-belge : la Castafiore, Gaston Lagaffe et Assurancetourix}

Les personnages de la Castafiore, de Gaston Lagaffe et d'Assurancetourix, sont créés respectivement en 1938 dans Le Petit Vingtième, I957 dans Spirou, et 1959 pour le lancement du journal Pilote. Leur rapport problématique à la musique les a rendus absolument emblématiques, tant leurs prestations, à l'image des sonorités du gaffophone, sont insupportables pour leur entourage. La cohérence thématique entre ces trois figures s'explique en partie par une même appartenance au genre comique, particulièrement enclin à renverser les hiérarchies en place pour le plus grand plaisir du lecteur. Et quelles cibles plus tentantes que l'art lyrique, l'orphisme romantique des odes celtes ou le raffinement courtois de la harpe, pour mener une entreprise burlesque de désacralisation?

Il est significatif que ces trois figures antimusicales se déploient en une période où la bande dessinée travaille à sa propre légitimation, et où se constitue le statut social de l'auteur, ce dont témoigne dès 1956 la première fronde des auteurs de bandes dessinées franco-belges contre les éditeurs. Tout de passe comme si la bande dessinée interrogeait les limites de son propre champ expressif, en se positionnant par rapport à d'autres médias. La musique fait figure à cet égard de grand Autre médiologique, que la $\mathrm{BD}$ ne pourra jamais rejoindre, quand bien même elle s'amuse à susciter l'illusion d'effets sonores, à travers des onomatopées, des symboles iconiques, ou des distorsions du lettrage. On comprend alors que ce soient des figures comiques plutôt ridicules, qui aient à charge de représenter cette altérité. Mais la mise à distance satirique, ou parodique, voisine ici avec un étrange effet de proximité. Ces formes d'expression, 
qu'elles soient hybrides comme l'opéra, ou supposées mineures, comme la chanson populaire dans Astérix, ou le jazz-rock dans Gaston, suscitent un deuxième effet réflexif qui interroge les fondements de la légitimation: qu'est-ce qui sépare un grand art lyrique tendant à se populariser par le microsillon, et des musiques populaires aspirant à la reconnaissance des cercles prescripteurs? Autant de questions qui touchent aussi la bande dessinée, dans ses rapports à la "grande» littérature, ou au cinéma. Se moquer des effets mélodramatiques de l'opéra, jouer de connivence avec le patrimoine chansonnier, ou faire cause commune avec des musiques marginales jugées dissonantes, n'est donc pas anodin quant au positionnement du médium dans le champ social de l'art.

Un troisième niveau réflexif, enfin, touche le pouvoir intrinsèque du médium dans son rapport à l'indicible. Si la musique a la capacité de mobiliser les corps et de soulever les âmes, ou de faire résonner l'humanité de l'homme dans ce qu' elle a de plus trouble et de plus ambivalent, qu'en est-il du pouvoir intrinsèque de la bande dessinée, quant à ses effets esthétiques, politiques et éthiques? Si la musique ne saurait plus se poser comme un substitut à la métaphysique, la bande dessinée n'aurait-elle a fortiori qu'une valeur mystifiante, pour l'ère postmoderne qui la voit naitre comme art? C'est ce dernier point que j'aimerais interroger ici, en faisant l'hypothèse que le thème musical permet au contraire au médium BD d'approfondir son rapport à l'indicible, voire au sacré. Par-delà les réactions viscérales qu'ils suscitent, on peut se demander en effet quelle chose inouïe Assurancetourix, la Castafiore et Gaston Lagaffe font littéralement entendre. Cette musique fait-elle résonner à l'intérieur de soi l'attirance pour le désir de la perte? Ou bien dit-elle quelque chose de la perte déchirante du désir? Par-delà l'examen des sources qui ont conduit à la création de ces figures, puis l'analyse des modalités castratrices de leur musique, je tenterai de frayer quelques pistes d'interprétation à caractère psychanalytique, avant d'interroger la portée politique et sociale de ces mises en scène. Il se pourrait bien alors que le mouvement de désacralisation inhérent au comique, s'inverse ici en la consécration de quelques valeurs irréductibles.

\section{Les figures et leurs sources}

La Castafiore apparaît pour la première fois en 1938 (en noir et blanc) dans Tintin en Syldavie. Poursuivi sur une route, Tintin fait signe à une voiture qui s'arrête. À l'intérieur, se trouvent Bianca Castafiore, de la Scala de Milan, et son accompagnateur qui ne s'appelle pas encore Igor Wagner. La cantatrice propose aussitôt à Tintin de l'écouter, et pour la première fois elle se met à 
chanter cet "air des bijoux» qui la rendra célèbre, tiré de Faust, l'opéra de Gounod. La prestation de la Castafiore fait fuir les animaux de la forêt, mais aussi Tintin qui prend le premier prétexte pour quitter la voiture. À la fin des années 20, la bourgeoisie belge d'expression française raffole de Gounod. Les contemporains de Hergé fredonnent la cavatine de Faust, "salut, demeure chaste et pure», rendue populaire par l'avènement du microsillon et de la radio. Des années 30 aux années 6o, les Marguerites belges se succèdent au Théâtre royal de la Monnaie de Bruxelles : Juliette Marsanne durant la saison 1933-1934, Claudine Boons de 1938 à 1945 et Huberte Vecray de 1949 à 1959. Rançon du succès : Faust fut tourné en dérision, et Hergé, qui n'aimait pas l'art lyrique, ne manqua pas de faire partie des détracteurs'. En 1947, dans la première version colorisée et rebaptisée Le Sceptre d'Ottokar, Hergé accentue le ridicule de son personnage et l'affuble d'une tenue de bourgeoise allemande début Renaissance, dont l'authenticité interdit malheureusement l'harmonie. Ses minauderies atteignent le sommet du saugrenu, rompant l'illusion théâtrale. Son organe fait vibrer le micro sur pied au point d'inquiéter un contrebassiste de la fosse. Sont ainsi posés les fondements comiques de ce personnage de dondon casse-tympan. En 1943, dans Les 7 Boules de cristal, c'est Milou qui hurlera à la mort devant la prestation de la diva.

Assurancetourix, quant à lui, est une parodie burlesque de l'image traditionnelle du barde celtique. C'est à la fin du XVIII ${ }^{\mathrm{e}}$ siècle, avec l'extraordinaire succès de la légende d'Ossian, publiée par MacPherson à Édimbourg en 1765 , que la figure du barde prend véritablement son essor ${ }^{2}$. En I8I3, Ingres peint pour la chambre de Napoléon au Quirinal un Songe d'Ossian, évocation grandiose avec le vieux barde au premier plan. Est ainsi fixée l'image mystique de l'aède celtique dont le chant visionnaire consacre la postérité et voisine avec la temporalité des dieux. Dans son Histoire de France, l'historien Henri Martin qualifie les bardes de "poètes héroïques et religieux, dépositaires des traditions nationales, qui célèbrent les grandes âmes ${ }^{3}$ ». La figure du barde prend ainsi place dans les manuels scolaires, au côté du druide gaulois. Or, quel contraste entre la sublimation poétique des héros de l'épopée celte et le sort bien connu réservé à Assurancetourix dès qu'il ouvre la bouche! Le renversement est d'autant plus burlesque que toutes les compositions du barde sont des parodies de chansons françaises à succès, qu'elles soient folkloriques ou de variété. Assurancetourix fait ainsi figure de génie incompris, en avance d'une vingtaine de siècles sur son temps. Impossible bien entendu de conférer

I. N. Sadoul, Entretiens avec Hergé, Casterman, 1989, p. 46-47.

2. Voir E. Hobsbawn et T. Ranger, The Invention of Traditions, Cambridge University Press, I983.

3. H. Martin, Histoire de France [I837], 5 éd., t. I, I865, p. 66. 
au chant de l'aède un quelconque contenu spirituel, religieux ou initiatique : les parodies des succès populaires de Sheila, Maurice Chevalier, Antoine ou Dalida se prêtent peu à la quête du sacré. La désacralisation burlesque du barde s'interprète alors dans un sens égalitaire et démocratique. Le village met à l'écart tout culte de la personnalité et tout esprit de sérieux, au profit de l'égalité conviviale et bon enfant des conditions. Dès lors qu'il renonce à exercer son art, Assurancetourix se rend cependant utile en faisant la classe aux enfants, ou encore en arbitrant un débat électoral entre Abraracourcix et Orthopédix. L'intellectuel est finalement accepté lorsqu'il se rend socialement utile par ses fonctions d'enseignant ou de journaliste. Mais qu'il ne vienne pas se faire le représentant d'un art élitiste, ni le dépositaire du secret des dieux. Avec son rationalisme laïc et démocratique, la série Astérix apparaît à cet égard fortement imprégnée par les valeurs du radicalisme de la III République ${ }^{4}$.

Avant d'inventer le gaffophone, Gaston Lagaffe s'essaye à de nombreux instruments : guitare, trombone, bombardon, scie, violon tzigane, klaxophone, guitare émettrice, tuba basse, avec une certaine prédilection pour le rock, et le jazz, des musiques encore marginales, à l'orée des années 60, qui de préférence font du bruit et dérangent l'ordre établi. Derrière cette thématique libertaire, on retrouve l'influence d'Yvan Delporte, le rédacteur en chef de Spirou de 1956 à 1968, qui joue dès 1957 un rôle très important dans la conception et la caractérisation du personnage. C'est souvent lui qui introduit la thématique musicale, à travers des idées de gag et de nombreux récits illustrés comme "Sifflez en travaillant", "Pouet pouet et paysan", ou "Pont des soupirs». Cette surenchère débouche sur l'invention du fameux gaffophone dans le gag $\mathrm{n}^{\circ} 449$ du 9 mars 1967. La moindre vibration produit une déflagration universelle à laquelle rien ne résiste. Toute la symbolique de raffinement courtois et de spiritualité que peut avoir la harpe celtique, est renversée ici en un objet grossier, primitif, quasi préhistorique : «un brontausorophone» dit Fantasio. L'objet a une connotation phallique évidente : une queue recourbée, une étrange forme de transept, qui peut faire penser à une croix chrétienne déformée, avec deux excroissances latérales recouvertes de peau de tambour, comme deux testicules géants, ou comme les deux bras coupés d'un tronc humain. Le socle, une sorte de tronc d'arbre évidé, évoque quant à lui l'orifice d'une corne d'abondance. Lorsqu'il s'aventure à trimballer son gaffophone dans la nuit $\left(8,27^{5}\right)$, en passant son corps à l'intérieur, Gaston ressemble à un monstrueux phallus sur pattes. La

4. Voir N. Rouvière, Astérix ou la parodie des identités, Flammarion, coll. «Champs», 2008, p. 68-74.

5. L'édition prise ici pour référence est l'édition Dupuis définitive qui suit l'ordre chronologique de l'œuvre. Le premier chiffre indique le numéro de l'album, le second indique le numéro de la page. 
scène d'horreur contient quelques réminiscences de Jack l'éventreur, dans les brouillards de Londres et la gent féminine est particulièrement terrorisée.

\section{La musique castratrice}

La musique de ces trois personnages a donc pour point commun de provoquer dans la fiction un effet de castration, qui touche les registres de l'imaginaire, du symbolique et du réel.

Dans le cas de la Castafiore, il faut concéder que l' «air des bijoux» n'a pas un effet horrifiant sur tout le monde. La Castafiore est tout de même de la Scala de Milan, c'est-à-dire sous contrat, au saint des saints de l'art lyrique. La cristallisation négative concerne en fait Tintin et le capitaine Haddock. Chez le jeune reporter, l' "air des bijoux» est tout bonnement générateur de phobie. Dans Le Sceptre d'Ottokar, il se cramponne en prison à la couverture de sa couche, et dans Tintin au pays de l'or noir (1950), change frénétiquement de longueur d'onde, lorsqu'il tombe sur une retransmission du fameux air. Haddock, dans Les 7 Boules de cristal, aborde quant à lui la prestation pour la seizième fois consécutive sans appréhension aucune. Sa première rencontre avec Bianca a lieu dans L'Affaire Tournesol dans les coulisses de l'opéra de Szohôd. Fasciné, bouche bée, Haddock bafouille son nom : «Hadada» qu'on peut lire «à dada». Cette manière enfantine de demander à jouer à cheval peut également s'interpréter comme une invitation sexuelle faite sur un mode ludique et puéril. Pour lui montrer qu'elle a reçu le message inconscient, la Castafiore l'appelle aussitôt monsieur Paddock, terme qui désigne à la fois un enclos aménagé pour les juments poulinières et un lit en langage populaire. À partir de cet instant, la Castafiore ne va cesser de déformer le nom du capitaine et de délivrer des messages étrangement érotisés. Dans Les Bijoux de la Castafiore, sa présence engendre une série d'incidents : pipe qui tombe, doigt puis appendice nasal mordus par un perroquet, piqûre de guêpe. L'angoisse de castration du capitaine Haddock se traduit par un cauchemar, au centre duquel se trouve l'"air des bijoux»: le capitaine s'imagine dans un théâtre, assistant au récital de Bianca. Il est assis au premier rang, tout nu; son visage est rouge, couleur de la honte et de l'érection. Sa nudité contraste avec celle des autres spectateurs, des perroquets en habit de gala, qui jugent sévèrement l'indécence du capitaine. Jean-Marie Apostolidès y voit un rappel des perroquets qui avaient conservé, dans Le Secret de la licorne, la tradition du chevalier François, l'ancêtre de Haddock, en se transmettant son répertoire d'injures $^{6}$. Le regard des oiseaux redirait ainsi la loi : le désir sexuel doit être

6. J.-M. Apostolidès, Les Métamorphoses de Tintin, Flammarion, coll. «Champs», 2006, p. 368370. 
réprimé. S'il se manifeste trop ostensiblement, les perroquets-juges puniront le coupable en le castrant. Bianca, qui chante l' «air des bijoux", a elle aussi une tête de perroquet, ce qui en ferait une femme réservée aux perroquets de la salle, les représentants de l'ancêtre. Selon le critique, le lit où dort le capitaine peut être interprété soit comme séparé du rêve, soit comme étant sur le plateau et faisant partie intégrale du numéro. Or cette mise en scène de la nuit de noce est en même temps une castration du mâle, car le chant est en fait un cri terrifiant, celui de la Castafiore, mais aussi, un peu plus haut dans l'album, celui de Miarka, la bohémienne qui mord le doigt du capitaine. La cantatrice se change ainsi en castratrice, femme interdite, selon Jean-Marie Apostolidès, parce qu'elle est réservée au père ou à l'ancêtre.

Avec Assurancetourix la castration quitte le registre de l'imaginaire pour celui du symbolique. Dans Astérix et les Normands, le barde donne un récital devant les guerriers nordiques, qui se mettent alors à trembler de tous leurs membres et à crier de peur, devant l'horreur innommable de sa voix. Le barde a modifié leur appréhension du corps, car au début de l'aventure, ils ne connaissent pas la peur. Une vignette l'illustre de façon comique. Deux savants normands se livrent à une étrange expérience : l'un tape sur la tête de l'autre à grands coups de massue et l'enfonce dans la neige comme un vulgaire pieu en bois.

- Alors?

- Rien. Tape encore. J'ai toujours plus de mal que de peur ${ }^{7}$.

L'inversion comique de l'expression «avoir plus de peur que de mal» montre ici que le lien au corps est une réification opaque. La matière corporelle ne parvient pas à faire image, pour déclencher l'appréhension de la souffrance subie. À l'image du guerrier planté droit dans le sol, les Normands sont les hommes de l'indivision, ils sont indivis, incapables de décoller de la sensation physique éprouvée. Persuadé que «la peur donne des ailes» et permet de voler, le chef Grossebaf décide d'entreprendre un "voyage d'étude» en Gaule, pour découvrir ce sentiment inconnu qu'est la peur. L’adage bien connu signifie que l'angoisse provoquée par la perspective d'un danger peut entraîner une réaction telle, que le corps ne pèse plus, qu'une limite physique est dépassée. L'imagination épouvantée de la menace l'emporte sur la stricte sensation physique de l'effort. Bien évidemment, il ne s'agit en aucun cas de battre des ailes comme les oiseaux. Mais la méprise des Normands est éclairante sur l'insu de leur désir. Tout se passe comme s'ils souhaitaient décoller de la matérialité brute, accéder à une forme de décorporalisation, pour que le physique prenne mentalement le statut d'image. Croire naïvement que

7. Goscinny et Uderzo, Astérix et les Normands, Dargaud, I966, p. 9, c. 7. 
«la peur donne des ailes» n'est donc pas insensé : il s'agit ni plus ni moins de sortir de la barbarie, en rompant avec l'opacité corporelle.

Arrivés en Gaule, ils capturent le Gaulois Goudurix, mais restent perplexes devant ses tremblements de peur. Ils tentent de les expliquer par des facteurs physiques extérieurs : le froid, la fièvre des marais, ou encore la grippe. Enferrés dans une vision strictement biologique, les Normands ne peuvent concevoir que le corps puisse être atteint par le mental. C'est le chant du barde, qui va provoquer ce décollement, en leur faisant éprouver à distance de fortes sensations physiques, tout en les plaçant devant une aporie du langage pour en rendre compte. Cette audition inouïe constitue une expérience déchirante : les Normands décollent de la corporalité brute, en se percevant pour la première fois de l'extérieur, à l'image du chant d'un autre. Ils se jettent aussitôt dans le vide et tentent de battre des ailes pour voler. Cette méprise comique dit aussi une vérité : l'accès du corps au statut d'image était désiré comme un décollage de soi. Les Normands ont ainsi accompli un chemin d'humanisation. Contrairement à Narcisse, qui dans la fable d'Ovide, s'évertue à abolir la frontière qui sépare son corps de son image, ils ont entrepris de déléguer le corps dans la représentation, pour rompre avec la corporalité brute et s'appréhender comme séparés d'eux-mêmes. Le chant du barde leur a fait intégrer le principe de la division subjective, la plausibilité même de la perte, de l'absence à soi-même.

Si l'on se place du point de vue des protagonistes de la fiction, la castration, Avec Gaston Lagaffe, passe dans le registre du réel. La sensation qui est en jeu est moins celle de l'ouïe que du toucher : tout part à chaque fois d'un léger effleurement, celle d'une branche de sapin dans les cordes, ou d'un souffle d'air. Le moindre frôlement des cordes du gaffophone fait tomber tout objet contondant ou pointu, à caractère phallique : quand ce n'est pas une cheminée d'usine qui s'écroule $(8,29)$, ou la maquette du nouvel immeuble Spirou $(9,3)$, ce sont les tuyauteries qui se dessoudent (II, 27), les aiguilles de sapin qui chutent brutalement (IO, IO), ou les avions de chasse qui menacent de s'écraser $(8,46)$. Plus emblématique encore, la musique de Gaston a pour effet de faire retomber le désir de Moiselle Jeanne. Sa jolie queue-de-cheval virevoltante retombe d'un seul coup en une masse chevelue informe ( 7, I2). La scène est à mettre en parallèle avec le gag où un baiser de Gaston électrise la secrétaire de désir, au point que sa chevelure s'emmêle dans le bouquet de gui suspendu au-dessus de sa tête. La scène la plus emblématique est sans doute la tentative de Fantasio de sectionner les cordes du gaffophone, afin d'éviter que la moindre vibration ne brise le service à porcelaine de $\mathrm{M}$. Dupuis $(8,25)$. Contre toute attente l'arc se déploie soudain avec une violence inouie et brise les caisses du service. C'est sans doute la représentation la plus forte 
de l'action castratrice. Un phallus se tend à l'extrême et brise les «bijoux» de l'éditeur.

\section{L'expression de l'inconscient}

Comment expliquer de tels effets castrateurs chez ces figures antimusicales? Le détour par la psychanalyse peut sans doute s'avérer éclairant, s'il est vrai, comme le note Serge Tisseron, que la $\mathrm{BD}$ est tout particulièrement propice à l'expression de mécanismes inconscients et facilite le retour régressif d'images mnésiques enracinées dans le trésor de l'enfance ${ }^{8}$.

De fait, les interprétations psychanalytiques ne manquent pas, pour expliquer la portée de l'" air des bijoux" dans Tintin. De petite-bourgeoise qu'elle était dans l'opéra de Gounod, Marguerite, est transformée par le don des bijoux en "fille d'un roi qu' on salue au passage». Elle rompt avec sa famille et pense vivre avec Faust, qu'elle prend pour un jeune seigneur, plus conforme à ses aspirations nobiliaires. Or, si la Castafiore fait de cet air son hymne personnel, c'est qu'elle s'identifie totalement à cette histoire.

Dans Tintin et le secret d'Hergé, Serge Tissseron a montré comment le scénario de la Marguerite séduite et abandonnée pouvait trouver sa source dans un secret de famille lié à la situation de Marie Dewigne, la grand-mère paternelle de Hergé employée chez la comtesse de Duzeele9. Marie Dewigne était fille-mère de deux jumeaux, qui ont porté pendant onze ans le nom de «Dewigne», et ont bénéficié de la générosité de la comtesse, avant d'être légitimés grâce à un mariage blanc, par un ouvrier du nom de "Rémi ». L'identité du grand-père secret n’a jamais été révélée. Dans le fantasme de Hergé, Marie Dewigne, tout comme la Marguerite de Faust, a pu s'éprendre d'un homme d'une condition sociale supérieure, accepter sa séduction et tomber enceinte en dehors de tout lien marital. Le travail de Benoît Peeters, autour de l'album Les Bijoux de la Castafiore, aiguille sur une hypothèse complémentaire, celle de la chaste fleur innocente et abusée ${ }^{\mathrm{ro}}$. Il met au jour une importante thématique sexuelle centrée sur la question de la virginité et questionne la qualité de l'événement qui recouvre le vrai-faux vol des bijoux. La Castafiore - ou Marie Dewigne a-t-elle été victime d'un vol ou d'un viol? Le fait que ce soit deux jumeaux, les Dupond eux-mêmes, qui soulèvent cette hypothèse par un lapsus, semble en effet une étrange coïncidence. Jean-Marie Apostolidès note lui-même que de façon significative, la Castafiore s'évanouit à chaque fois

8. S. Tisseron, Psychanalyse de la bande dessinée, Flammarion, coll. "Champs», 2000.

9. Id., Tintin et le secret d'Hergé, Hors collection / Presses de la Cité, 1993.

Io. B. Peeters, Lire Tintin - Les Bijoux ravis, Les Impressions nouvelles, 2007, p. 156-169. 
qu'elle voit un homme s'introduire apparemment avec brutalité dans un lieu où elle se trouve ${ }^{\text {II. }}$ C'est le cas dans Le Sceptre d'Ottokar lorsque Tintin pénètre par effraction dans la salle du palais, et la scène se reproduit à l'identique au château de Moulinsart, après le pseudo-vol des bijoux. Un peu plus tard elle pousse un cri d'horreur dans la nuit, à cause d'une ombre, le fantôme d'un grand-duc, un "monstre dont les yeux brillaient comme des diamants" et qu'elle associe aux "pas d'un homme, sans aucun doute». Elle semble associer ces effractions à une tentative de vol ou de viol sur sa personne. Serge Tisseron évoque une troisième hypothèse, où les jumeaux seraient les enfants non reconnus de la comtesse, nés de parents nobles, et confiés à la garde de la servante Marie Dewigne. Irma, la servante de la Castafiore, aussi muette que sa maittresse parle pour ne rien dire, est du reste suspectée par les Dupondt d'avoir volé les bijoux de sa maîtresse, c'est-à-dire, dans l'analogie enfants-bijoux, d'avoir dérobé ses jumeaux. Notons que le prénom Irma est une anagramme, par inversion des syllabes, de celui de Marie. Qu'il s'agisse d'une mère illustre ayant abandonné ses enfants à la garde d'une servante, d'une femme de maison abusée par le maitre, ou d'une malheureuse ayant élevé seule ses enfants, la Castafiore condenserait les différents scénarios que Hergé aurait nourris à propos de sa grand-mère, porteuse du secret initial qui marqua le destin de la famille Dewigne, puis Rémi. D'où sans doute l'impossibilité de la cantatrice à donner un nom au capitaine Haddock, dont Hergé a dit à maintes reprises qu'il le représentait lui-même. On comprend également qu'elle ne puisse pas verbaliser sa sexualité autrement que sous forme de lapsus, de distorsions phonétiques et d'inversions. Dans ce contexte, l'«air des bijoux" interprété par la Castafiore constitue pour les protagonistes de Moulinsart un retour du refoulé aussi sidérant qu'effrayant.

Le motif du gaffophone pourrait bien être propice lui aussi à l'expression de mécanismes inconscients trouvant à se représenter dans le corps de l'image, et alimentant la "pulsion scopique» du lecteur. La musique à corde de Gaston est l'unique événement, dans l'ensemble de la série, qui fait retomber le désir de Moiselle Jeanne. Il existe cependant une exception significative : l'aspect monstrueux de notre garçon de bureau, lorsqu'il est recouvert d'aiguilles de sapin de la tête aux pieds, après un accident provoqué par sa machine à projeter des aiguilles sur des carcasses de résineux $(9,8)$. Devant la fourrure d'aiguille ambulante, qu'est devenu le jeune homme, Moiselle Jeanne pousse un cri d'horreur. Gaston, déprimé, décide alors de trouver un peu de consolation dans la musique, et ce sont les vibrations du gaffophone qui vont finalement le libérer de son martyre, en faisant tomber les aiguilles

II. Op. cit., p. 382-383. 
(9, Io). Rejouer sous une forme détournée l'effet négatif permet donc ici de retrouver la situation positive de départ. Ce schéma d'une libération par la musique contiendrait-il l'une des clés du rôle assigné au gaffophone? Premier temps : l'envie de redonner sa verdeur et sa vigueur à un squelette végétal, qui suscite la compassion de Gaston. Second temps : par déplacement, c'est Gaston qui reçoit la projection, signe que c'est peut-être lui, dont la verdeur et la vigueur étaient abîmées. En se transformant en gaffe, l'action qui était censée régénérer dévoile alors peut-être malgré elle la vérité de la blessure et sa cause : la souffrance d'être fourré lorsque l'on est encore vert. Être fourré : c'est-à-dire être un petit massif d'épines de taille moyenne, mais aussi être couvert et pénétré à la fois. Le résultat en tout cas est dévastateur pour l'image de soi et le désir de l'autre. Notons que l'effet du gaffophone n'est jamais aussi monstrueux que lorsque le corps de Gaston est fourré dans l'orifice : c'est le cas auprès des passants, lorsque Gaston transporte le gaffophone dans la nuit, ou lorsqu'il se trouve introduit par Fantasio comme sourdine dans le corps de l'instrument. Faisons alors une hypothèse : les gags à répétition mettant en scène les destructions causées par la musique du gaffophone, sont peut-être la représentation inversée d'une autre scène, où le sujet serait objet d'attouchement et de pénétration. Et c'est peut-être ce trauma destructeur que le gaffophone fait entendre, en provoquant un tel ébranlement de la nature et de l'ordre du monde.

Une telle hypothèse est sans doute à mettre en lien avec une autre série de gags à caractère tout aussi obsédant et obsessionnel : celui de la signature des contrats que Gaston fait toujours échouer. Pierre Glaudes y voit une représentation altérée de ce que la doctrine freudienne appelle l'Urszene $e^{12}$. Il s'agit d'une scène représentant les rapports sexuels entre les parents, tels qu'ils sont observés par l'enfant qui les comprend comme une agression du père dans une relation sadomasochiste. Celui qui incarne le rôle du père, ici c'est Demesmaeker, dont le nom n'est autre que le patronyme d'un collaborateur de Franquin, Jidéhem, Jean de Mesmaeker, depuis le jour où ce dernier s'est exclamé «il ressemble à mon père!» en voyant le personnage de l'homme d'affaires. Franquin a trouvé cette idée excellente et a demandé à Jidéhem de l'autoriser à reprendre son patronyme. Fantasio, qui perd totalement sa fantaisie des débuts au contact de Gaston, et plus encore Prunelle, dont le nom évoque à la fois la présence obsédante du regard et la petite prune à croquer, représenteraient selon Pierre Glaudes le corps maternel, la maison Dupuis, dont le critique note qu'elle suggère des profondeurs aquatiques. Le

I2. P. Glaudes, "Signerai jamais ces contrats! - Notes sur le rire dans Gaston Lagaffe», Lendemains, $\mathrm{n}^{\circ}$ 50, 1988 , p. 7-15. 
scénario consisterait alors de façon immuable à empêcher l'action du père, à renverser son ordre, avec cette originalité que la transgression n'est marquée ici par aucun sentiment de culpabilité, mais par une affirmation du principe de plaisir et une libération pulsionnelle, offrant au lecteur la satisfaction d'une régression pour rire.

Si la scène des contrats a effectivement une connotation sexuelle, il semble difficile en revanche de situer la figure maternelle. Prunelle est le seul personnage de la série affublé d'une barbe, et son nom féminin dit plutôt la castration. De fait l'ordre paternel, dont il est le serviteur est chez lui davantage chargé d'angoisse, d'où sans doute la référence obsédante au regard. Ajoutons que Demesmaeker appartient pour Prunelle à la génération des aînés. On sait que la fille cadette de l'homme d'affaires roule en décapotable, ce qui donne approximativement à l'homme d'affaires 45 à 50 ans, entre Io et 20 de plus que les membres de la rédaction. Si scène sexuelle il y a, c'est entre deux personnes de sexe masculin, et de deux catégories d'âge différentes. Notons par ailleurs que les interventions de Gaston ne sont pas anodines; elles dévoilent toujours chez Demesmaeker une double nature. Ce dernier est régulièrement associé à une figure de monstre, d'animal à cornes (vache, cerf et surtout rhinocéros), à une figure d'ogre (qui mange ses contrats ou les fait manger), ou de transgresseur de la loi (il se fait souvent verbaliser pour des infractions supposées au code de la route). Autrement dit Gaston révèle la dimension contre-nature, sauvage et dangereusement transgressive du contrat qui se trame. Le gag à répétition pourrait bien être alors la représentation détournée d'une autre scène, où l'humiliation infligée à Demesmaeker (pénétration par des griffes, des piques, des épines, ou castration visant le nez, le pied, le cigare) serait le renversement d'une humiliation subie dans la réception d'un bureau. La connotation religieuse présente dans la première apparition du personnage $(2,36)$ ainsi que dans son nom ("de messe m'écœure») pourrait faire penser au père d'une institution religieuse. Si cette interprétation se confirme, le gaffophone donnerait à entendre de façon détournée la déflagration que représente tout geste d'attouchement dans un tel contexte.

Le détour par l'hypothèse psychanalytique permet me semble-t-il de mieux appréhender la portée politique et sociale que les auteurs donnent à ces figures antimusicales, dans une logique de dépassement, comme dans Tintin, ou au contraire d'extension, dans le cas de Gaston Lagaffe. 


\section{La musique comme pouvoir de résistance}

Au fil des albums, le personnage de la Castafiore évolue positivement et perd peu à peu son caractère de dondon casse-tympan. Dans L'Affaire Tournesol, elle a remis la plus belle robe de Marguerite pour accueillir ses admirateurs. Mais cette fois-ci, contrairement à l'allure ridicule qui était la sienne dans Le Sceptre d'Ottokar, elle est tout bonnement somptueuse dans son costume écarlate. Elle a abandonné ses nattes au profit de deux macarons, porte son corsage avec beaucoup de naturel et maîtrise l'ampleur de sa cotte par un gracieux mouvement du poignet. Elle déploie tact et bienveillance pour mettre tout son petit monde à l'aise, et risque sa vie pour sauver celle de Tintin et du capitaine. Dans Coke en Stock elle est exquise dans sa silhouette à cerceaux bleu et rose Pompadour. Sa joie de vivre est communicative et déteint sur Tintin lui-même qui sourit, comme s'il était en passe de guérir de son aversion pathologique des débuts. Son contre-modèle, comme le note Mireille Moons ${ }^{13}$, est sans doute Peggy, la colombe du général Alcazar dans Tintin et les Picaros, une épouvantable harpie qui incarne tout ce que Hergé déteste : le laisser-aller vestimentaire, l'impudeur, le m'as-tu-vu et surtout la dictature domestique. A contrario, rien n'a raison de l'éclat de la Castafiore : ni le décalage horaire, ni les geôles tapioquistes. Triomphante à sa descente d'avion en capeline et tailleur roses, pendants d'oreilles et bracelets assortis, elle le reste au cachot. Lors de son procès télévisé en direct, dans la grande salle du palais de justice de Tapiocapolis, elle est calme et éblouissante de témérité :

La prison à vie? Ai-je bien entendu?... Mais vous êtes grotesque, militaire! ou alors fou à lier, mon pauvre ami! Vos documents irréfutables! FFFT... Fabriqués de toutes pièces! j'en ris moi, moi de ces documents... Oui, j'en ris, parfaitement... ${ }^{\text {I4 }}$

Malgré les objurgations de ses juges qui tentent désespérément de la faire taire, la Castafiore entonne alors en plein prétoire le "grand air des bijoux". Face à ce chant de résistance, la retransmission télévisée est interrompue. Le rossignol cloue le bec au mensonge, réduit le totalitarisme au silence. À ce stade, son chant n'incarne plus le narcissisme maléfique, ni la fascination pour le désir de se perdre, mais exprime bien plutôt un individualisme héroïque, où le miroir est un gage irréductible d'indépendance. En somme, Bianca Castafiore n'est pas une diva tyrannique et hystérique, comme on a pu hâtivement la

13. Voir M. Moons, Bianca Castafiore - La diva du vingtième siècle, Éditions Moulinsart, 2006.

I4. Hergé, Tintin et les Picaros, Casterman, 1976, p. 48, c. 2-4. 
caricaturer, c'est une femme de tête dynamique et moderne, certes rigoureuse avec ses collaborateurs, mais qui prend aussi leur défense avec courage, comme dans Les Bijoux de la Castafiore, lorsque Irma est injustement accusée de vol. C'est une patronne, une chef d'entreprise exigeante qui veille sur son image de marque comme sur son unique produit de fabrication. Elle se positionne ainsi comme femme à part entière. Et, si elle dérange les autres jusqu'à l'exaspération, elle a aussi le talent de faire évoluer leur relation à autrui, ce qui est pour le moins positif dans le cas des trois vieux garçons de Moulinsart. Ce personnage antimusical débouche ainsi sur une figure féministe exemplaire de la défense des libertés individuelles.

À travers le gaffophone, Franquin, quant à lui, fait résonner sans concession le credo de la résistance libertaire et anarchiste. Pour cerner la portée de la mise en scène, il faut reconsidérer la violence avec laquelle l'arc de l'instrument brise les porcelaines de $\mathrm{M}$. Dupuis, suite à la section des cordes $(8,25)$. Difficile d'imaginer auparavant une telle tension dans la courbure de la branche. A posteriori, "le boulot du tonnerre» que selon ses propres mots, Gaston a dû fournir pour parvenir à plier l'arc, semble absolument démesuré. Le gaffophone fait ainsi retentir l'inouï : la souffrance qu'il y a à se plier, à ployer, deux verbes qui sont étymologiquement liés à «emploi» et «employer». Se plier signifie pour Gaston commencer par se tenir droit, l'un des premiers objectifs que Fantasio lui assigne. Mais tel est pris qui croyait prendre. Gaston lui assène un air de violon tzigane qui contracte de la tête au pied son supérieur, «toujours trop tendu» $(6,22)$. Les gags à répétition du Gaston en latex, cette poupée molle que l'on peut plier en tous sens et que l'on confond avec le protagoniste, sont sans doute une manière de signaler la tromperie des apparences. S’il se confond avec une poupée pliable, en réalité Gaston ne cède jamais sur le terrain de son désir propre, pour lequel il est inflexible. Résister, a contrario, c'est se détendre, onduler. Le gaffophone fait vibrer et onduler tout ce qui est rectiligne et droit : le pont Ducran Lapoigne $(7,16)$, les avions de chasse $(8,46)$, et même les poteaux électriques, qui se mettent à danser $(8,47)$, ce qui rappelle les arabesques formées par la queue du marsupilami, symbole de liberté et de créativité. On ne s'étonnera donc pas que la musique de Gaston empêche de se plier au travail : à plusieurs reprises, elle provoque des sorties d'usine anticipées $(5,4 ; 6,22)$ et perturbe le quotidien de la rédaction du journal Spirou, au point d'entraîner des représailles de la part des employés. À cet égard le gag du gaffophone qui brise les porcelaines de M. Dupuis, se termine par une ellipse significative. Fantasio se retrouve à faire la manche sous un pont, assis à côté d'un clochard. On suppose qu'il a été limogé par l'éditeur et qu'il se retrouve sans emploi, injustement brisé dans son honneur. C'est un prolongement inattendu qui 
dévoile une représentation collective au cœur de l'idéologie du travail : à savoir qu'il n'y a de choix qu'entre l'emploi et la clochardise, prendre le pli ou se désocialiser. Fantasio est prisonnier de cette alternative moralisatrice, témoin d'un imaginaire collectif où le clochard est hors système, improductif par choix. En témoignent les sobriquets dont Fantasio affuble Gaston : ce parasite, ce cloporte, cet improductif, cet anormal, qui refuse précisément de se plier. Gaston serait un clochard d'un nouveau type, plus subversif encore, parce que hors système au cœur du système. De fait Gaston est celui à qui on demande constamment de se normaliser, de s'insérer par le travail. Or le gaffophone vient faire retentir l'inaudible, pour la petite musique sociale, en son point le plus névralgique, le plus vital : le refus de s'aliéner dans ce pli collectif. Dans son livre Les Naufragés, qu'il consacre aux sans-abri, Patrick Declerck montre combien les clochards payent l'assistance en monnaie symbolique, en montrant leur bonne volonté de réinsertion par le travail, mot fétiche au cœur de l'idéologie qui structure le traitement de la désocialisation ${ }^{15}$. Se produisent alors un transfert et un contre-transfert (du soignant sur le soigné) qui sont préjudiciables à la relation. Le clochard est sommé de se réinsérer, de se normaliser par le travail, alors qu'il en est justement incapable. L'attitude de Gaston, inflexible sur la question du désir, marque une revanche sur cette idéologie normative. Le paradoxe, c'est que Gaston est en réalité un inventeur d'une fécondité rare, pour ne pas dire un homo faber de génie. Mais c'est sans doute là toute la différence entre travailler, et faire œuvre. Dans La Condition de l'homme moderne, Hannah Arendt caractérise le travail comme une activité cyclique qui ne connaît jamais de fin, inféodée au processus biologique de subsistance. L'œuvre à l'inverse est un processus qui a un terme et s'achève dans un objet qui possède sa propre existence dans la durée, indépendamment de l'acte qui l'a produite. Or l'époque moderne ne permettrait plus de distinguer l'homo faber et l'animal laborans. La manière moderne de fabriquer les objets s'accomplirait sur le mode du travail, au profit d'un processus qui finit par expulser l'homme lui-même. La création de l'hebdomadaire Spirou est elle-même aliénée au mode du travail, avec ses horaires, ses délais, ses obligations contractuelles, et sa division rationnelle des tâches. Et quelle activité symboliquement plus cyclique, interminable et épuisante que de classer le courrier des lecteurs et d'y répondre? La réponse de Gaston à l'idéologie productiviste qui aliène l'œuvre par le travail, c'est d'aliéner le travail par l'œuvre : en créant des objets inutiles (machines à faire des ronds de fumée et autre mastigaston), en créant des objets auxiliaires qui en réalité parasitent ou sabotent la mécanique des tâches, ou en faisant

I5. P. Declerck, Les Naufragés : avec les clochards de Paris, Plon, $200 \mathrm{I}$. 
œuvre de loisir au cœur du processus productif (cuisine, jokari, musique, etc.). Ultime subversion et retournement des valeurs : l'invention du ressort sauteur qui parcourt la rédaction en tous sens, symbole du cycle indéfini et aliénant de la répétition, s'affiche comme l'invention «du mouvement perpétuel », œuvre immortelle par excellence, qui résiste au temps.

Au terme de ce parcours, la désacralisation de la figure du barde gaulois s'inverse quant à elle en un mouvement sacralisant. Dans l'économie politique du village, le barde constitue une référence légale à part entière, au même titre que le chef et le druide. La voix d'Assurancetourix a le pouvoir d'infliger la limite de ce qui est humainement supportable. Elle oblige les auditeurs à reconnaître qu'une instance de pouvoir les dépasse absolument. De façon significative, le personnage est qualifié d' «innommable» à la page 4 de chaque album, où sont présentés "quelques Gaulois» : autrement dit l'horreur inouïe de sa voix ouvre sur un abîme, quelque chose qui n'a pas de nom. Elle notifie une véritable impasse de la représentation. Ainsi le chef normand n'a pas de mot pour désigner ce qu'il entend, et ne peut qu'évoquer l'effet produit sur son corps :

À l'idée d'entendre ça encore une fois, mes genoux tremblent, mes dents claquent, des sueurs froides couvrent mon front et mon estomac se noue ${ }^{16}$ !

Les mots sont toujours en deçà de l'horreur inouïe de ce chant. Le ligotage, la baffe, la destruction de l'instrument ou le bâillon, ne font que rappeler l'existence de ce point de butée de la représentation langagière. Quelque chose se tait absolument derrière le langage et à travers lui. La parole n'existe peut-être même qu'au nom de ce vide qui la fonde, cet innommable qu'elle recouvre. La censure rituelle trace alors une ligne d' «inter-dit» : elle situe toute parole comme un dire d'interposition entre l'homme et l'abîme de l'indicible. Elle stipule que nul n'est transparent à son propre langage ni à soimême. Assurancetourix a ainsi valeur de totem pour le village. Il crée la mise en scène du vide, constitutif de la parole. Sous son égide, la communication peut battre son plein. Le rituel du bâillon fait surgir l'espace tiers de l'écart, la place qui tient l'être de parole indéfiniment à distance de soi et en retrait du monde, faisant ainsi obstacle à la scène narcissique. On comprend alors pourquoi Astérix inscrit le barde comme nouveau locataire dans le Domaine des dieux, le nouveau complexe urbanistique construit par les Romains à proximité du village. La campagne publicitaire orchestrée par le pouvoir impérial fait la promotion mensongère d'un homme nouveau menant une vie de plénitude heureuse, offrant l'accès à tous les plaisirs. Sur une double

I6. Op. cit., p. 43 , c. 9. 
page $^{17}$, les auteurs représentent la plaquette de marbre vantant les mérites de cette vie nouvelle exauçant tous les désirs du sujet roi :

VOUS AIMERIEZ VIVRE COMME UN DIEU ? ALORS... LE DOMAINE DES DIEUX EST

POUR VOUS !

UNE VIE SAINE ET HEUREUSE, DIGNE DE CELLE D'UN DIEU!

Cette vie rêvée est décrite sur le mode du plein, de l'absence de manque. L'homme vit comme un dieu, le monde n'existe que pour son plaisir. Aucune instance ne le dépasse. Que l'on ne s'y trompe pas : la critique des auteurs est politique et vise la société contemporaine. Dans Le Domaine des dieux, les «impliables» en marbre sont bien plus que des plaquettes publicitaires, ce sont des tables utopiques de la Loi, décrivant la vie de l'homme nouveau. Les auteurs pointent alors ce qui semble constituer le noyau fantasmatique du nouveau discours normatif : le slogan du client-roi cache la dangereuse utopie du sujet-roi, qui affirme le droit des individus à la jouissance pleine. En déclinant le rêve d'une absolue plénitude, où les moindres désirs peuvent être assouvis, la publicité escamote la référence à l'interdit, destinée à limiter chaque sujet. Elle semble au contraire cautionner un imaginaire narcissique, pour flatter le client. On conçoit ce qui oppose la figure du barde à ce fantasme de totalité. Assurancetourix donne accès à la représentation de la négativité pure. Chacun est mis à la place qui est la sienne et entre dans l'économie de la perte. À travers lui, l'ordre politique du village gaulois differe à jamais la jouissance, en notifiant le manque, l'absence, comme une nécessité structurale et logique $^{18}$. Les institutions villageoises font ainsi obstacle à la scène narcissique et au désir de toute puissance.

On l'aura compris, ces trois figures antimusicales, emblématiques de la bande dessinée franco-belge des années 50 à 70 , ne se réduisent pas à une pure entreprise de désacralisation. La trouée que constitue la représentation problématique de la musique, comme grand Autre médiologique, fonctionne au contraire, auprès des auteurs, comme un appel, pour faire résonner l'insu inouï de leur discours par la bande. En ce nouvel espace indicible, où s'entremêlent problématique individuelle et symbolique collective, se forge sans doute le pouvoir mythifiant du médium $\mathrm{BD}$, et son aptitude à dire quelque chose de la constitution anthropologique du sujet et des sociétés. Ainsi le bâillonnement d'Assurancetourix consacre l'innommable comme un lieu

17. Goscinny et Uderzo, Le Domaine des dieux, Dargaud, I971, p. 28-29.

I8. Voir N. Rouvière, Astérix ou les lumières de la civilisation, Presses universitaires de France, «Partage du savoir», 2006, p. 23-27 et p. 209-217. 
séparé, une extériorité radicale, irréductible. On retrouve là le sens étymologique du mot templum qui provient de la racine grecque temnô signifiant "couper, fendre, trancher», ce qui a donné temenos, "temple». Templum signifie littéralement «le lieu où se tient ce qui est coupé». À ce titre, l'utopie du village gaulois pourrait être qualifiée de société "sanctualisante», pour reprendre une expression forgée par Pierre Beckouche, dans un article qu'il consacre au symbolique ${ }^{19}$. Pierre Beckouche rappelle la distinction entre les termes sacer et sanctus chez les Romains. Le sacer est le sacré par essence, alors que le sanctus est le sacré par l'action des hommes. Dans le cas de la Castafiore, l' "air des bijoux" apparaît dans un premier temps comme une menace narcissique, faisant craindre au sujet le risque de s'abîmer dans la confusion des places généalogiques. Mais au terme d'une évolution du personnage, il en vient à consacrer le reflet de soi dans le miroir, comme une irréductible garantie d'identité, contre toute oppression du pouvoir. En tout point exemplaire par son indépendance, sa franchise et la persistance qui est la sienne dans son être propre, la Castafiore peut bien être qualifiée par Hergé de "divine» dans le rôle de Marguerite, en tant que figure de proue libératrice d'un nouvel âge de l'individualisme. Enfin, dans le cas de Gaston Lagaffe, la musique destructrice du gaffophone, qu' elle fasse entendre ou non la déflagration d'un trauma psychique, met à bas l'ordre du monde pour consacrer l'œuvre inutile et gratuite, comme garde-fou du désir individuel et de la liberté humaine.

19. P. Beckouche, "Le symbolique : une approche lacanienne pour les sciences sociales", Le Débat, $\mathrm{n}^{\circ}$ 126, sept.-oct. 2003, p. 174-19I. 
